\title{
MicroRNA-205-5p regulates extracellular matrix production in hyperplastic scars by targeting Smad2
}

\author{
JUN QI $^{1}$, YIFEI LIU ${ }^{2}$, KESU HU ${ }^{1}$, YI ZHANG ${ }^{1}$, YANGYANG WU ${ }^{1}$ and XIA ZHANG ${ }^{1}$ \\ Departments of ${ }^{1}$ Burn and Plastic Surgery and ${ }^{2}$ Pathology, Affiliated Hospital of Nantong University, \\ Nantong, Jiangsu 226001, P.R. China
}

Received July 27, 2017; Accepted January 3, 2019

DOI: $10.3892 /$ etm.2019.7187

\begin{abstract}
Hypertrophic scar (HS) formation is the result of poor skin-wound healing. At present, the pathogenesis of HS formation is largely unclear. Micro (miR)RNAs have important effects on a variety of biological and pathological processes. The role of miRNA in HS formation remains largely unclear. The present study aimed to investigate the role of miR-205-5p in HS, and explore the underlying molecular mechanism. Reverse transcription-quantitative polymerase chain reaction (RT-qPCR) was used to determine the expression of miR-205-5p in HS. Western blot assay and RT-qPCR were performed to assess the expression of associated proteins and genes, respectively. TargetScan was performed to predict the target gene of miR-205-5p, and the luciferase reporter assay was applied to verify the prediction. The function of miR-205-5p on cell proliferation was detected using Cell Counting Kit-8 assay, and cell apoptosis was detected via flow cytometry. miR-205-5p expression was decreased in HS tissues and human hypertrophic scar fibroblasts (hHSFs). Mothers against decapentaplegic homolog (Smad)2 was significantly increased in HS tissues and HSFs, and it was directly targeted by miR-205-5p. Restoration of miR-205-5p suppressed HSF cell proliferation and induced cell apoptosis. It was also demonstrated that RAC-Alpha Serine/Threonine-Protein Kinase (AKT) phosphorylation and the expression of $\alpha$-smooth muscle actin, collagen I and collagen III were inhibited by miR-205-5p. In addition, Smad2 weakened the effects of miR-205-5p on HSFs. In conclusion, miR-205-5p exhibited an important role in HS by targeting smad 2 and suppressing the AKT pathway. These findings provide a clearer understanding of the mechanism for HS that may be used to develop novel treatments for HS.
\end{abstract}

Correspondence to: Dr Jun Qi or Dr Yangyang Wu, Department of Burn and Plastic Surgery, Affiliated Hospital of Nantong University, 20 Xisi Street, Nantong, Jiangsu 226001, P.R. China

E-mail: qijun170727@163.com

E-mail:wuyy2017@163.com

Key words: microRNA, hypertrophic scar, Smad2, AKT

\section{Introduction}

Hypertrophic scar (HS) is characterized by excessive growth of dense fibrous tissue, which is caused by deep heat or traumatic injury of the skin (1-3). In humans, HS is hyperplastic healed fibroplastic diseases, which are procedural processes that include proliferation, maturation, inflammation, and remodeling (4). Presently, there have been several methods for treating HS, such as surgical resection, injection steroids, radiotherapy, but still no best regimen, and the clinical behavior of HS is unclear. Some studies have demonstrated that many different non-coding RNAs and growth factors are involved in the formation of HS $(5,6)$.

MicroRNAs (miRNAs) are evolutionary conserved non-coding RNAs of about 19-25 nucleotides, function by regulating one or more mRNA to regulate gene expression for translation inhibition or cleavage $(7,8)$. About one-third of the coding genes in mammalian are regulated by miRNAs $(9,10)$, and mature miRNAs are not transformed into proteins but are bound to mRNAs to interfere with the translation process. With regard to miRNA function, they play a key role in cell proliferation, cell death and organ development $(11,12)$. Furthermore, abnormal expression of microRNAs is associated with several pathological processes, including kidney, lung and heart metabolism (13). Previous study have shown that miRNAs contribute to HS or keloid formation, and the abnormal expressed miRNAs have been identified by genomic analysis between denatured dermis and normal skin, which means that multiple signaling pathways participate in wound healing (14). For example, microRNA-98 has been found can inhibit the cell proliferation of human hypertrophic scar fibroblasts via targeting Col1A1 (15). MicroRNA-185 plays critical roles in HS via regulating transforming growth factor- $\beta 1$ and collagen-1 expression, and may serve as a promising target for HS treatment (16). miR-21 has been recognized as a critical regulator for HS formation (17). Recently, the effect of miR-205-5p in carcinogenesis has been well documented, in which many targets of miR-205-5p have been defined in cancer cells (18-21), and the effect of miR-205-5p in HS formation remains unclear.

Increasing evidence have supported that miR-205-5p may play critical roles in cell proliferation, apoptosis, and extracellular matrix $(\mathrm{ECM})$ deposition $(22,23)$. And increased myofibroblasts and excessive ECM accumulation are the main 
characteristics of HS formation (24). Thus, we supposed that miR-205-5p may play critical roles in HS formation. In this study, we revealed the abnormal expression of miR-205-5p in HS. Meanwhile, miR-205-5p overexpression prevented HSF cell proliferation and induced apoptosis. Moreover, we found that Smad2 was a direct target for miR-205-5p in HSF cells. miR-205-5p might serve as a new potential therapeutic target for HS.

\section{Materials and methods}

Tissue samples. In total, 15 paired Hypertrophic scar (HS) (Age range: 21-49 years old; sex ratio: 1:1; Location: skin) and normal skin (NS) (Age range: 23-51 years old; sex ratio: 1:1; Location: skin) tissues were obtained during biopsy from 15 patients at the Affiliated Hospital of Nantong University. All tissues were immediately stored in liquid nitrogen until use. All the protocols were approved by the Ethics Committee of the Affiliated Hospital of Nantong University (Nantong, China), and every patient wrote the informed consent.

Cell culture. The human embryonic skin fibroblasts, CCC-ESF-1 (ESF), and human hypertrophic scar fibroblasts (HSFs) were cultured in RPMI-1640 medium supplemented with $10 \%$ fetal bovine serum and $1 \%$ penicillin/streptomycin. All cells were incubated in a humidified incubator at $37^{\circ} \mathrm{C}$ and $5 \% \mathrm{CO}_{2}$.

$R N A$ extraction and reverse transcription-quantitative polymerase chain reaction ( $R T-q P C R)$. Total RNA from cells and tissues was extracted by using TRIzol reagent (Invitrogen; Thermo Fisher Scientific, Inc., Waltham, MA, USA), and cDNAs were synthesized using miScript Reverse Transcription kit (Qiagen GmbH, Hilden, Germany) according to the manufacturer's instructions. The primers for reverse transcription and amplification of miR-205-5p and U6 were designed and synthesized by Guangzhou RiboBio Co., Ltd., Guangzhou, China. Quantitative real-time PCR was conducted to detect the miR-205-5p and smad2 mRNA using the SYBR Premix Ex Taq ${ }^{\mathrm{TM}}$ II (TliRNaseH Plus) kit (Takara Bio, Inc., Otsu, Japan) with the Bio-Rad machine (Bio-Rad Laboratories, Inc., Hercules, CA, USA). U6 small nuclear RNA and GAPDH were used as internal normalized references for miRNA and mRNA, respectively.

Cell transfection. miR-205-5p mimics, smad2-plasmid and matched negative control (NC) were obtained from Guangzhou RiboBio Co., Ltd. Cell transfections were performed using Lipofectamine 2000 (Invitrogen; Thermo Fisher Scientific, Inc.), according to the manufacturer's instruction. Cells were harvested for further experiments after $48 \mathrm{~h}$ transfection.

Cell proliferation assay. Cell Count Kit-8 assay (CCK-8, Dojindo Molecular Technologies, Inc., Kumamoto, Japan) was used as a qualitative marker for cell proliferation ability. After $48 \mathrm{~h}$ transfection with miR-205-5p mimics, NC or miR-205-5p mimic+smad2-plamid, HSF cells were seeded into 96 well plates in triplicate at $5 \times 10^{3}$ cells per well. At $24 \mathrm{~h}, 10 \mu \mathrm{l}$ of CCK-8 solution mixed with $90 \mu$ l of RPMI-1640 was added to each well. After $2 \mathrm{~h}$ of incubation, the absorbance was measured at $450 \mathrm{~nm}$.

Apoptosis assay. HSF cells were transfected with miR-205-5p mimics, NC or miR-205-5p mimic+smad2-plamid, and $48 \mathrm{~h}$ after transfection, the cells were then subjected to apoptosis assay. Then $10^{6}$ treated cells were stained with Annexin V/PI using an apoptosis detection kit (BD Biosciences, Franklin Lakes, NJ USA). According to the manufacturer's instructions, after incubating for $15 \mathrm{~min}$ in the dark, cell apoptosis was then detected by flow cytometry.

Luciferase reporter assay. For confirmation of direct target binding, the wild type ( $\operatorname{smad} 2 \mathrm{WT})$ and mutant $(\operatorname{smad} 2 \mathrm{MUT})$ 3'UTR of smad 2 identified by TargetScan were cloned into a pmiR-RB-ReportTM dual luciferase Reporter gene plasmid vector (Guangzhou RiboBio Co., Ltd.). The UTR region of candidate target gene was inserted downstream of the sequence of Renilla luciferase, which was designed for reporter fluorescence (Rluc). For luciferase reporter analysis, luciferase reporter vectors and mimic control, miR-205-5p mimics, were transfected into HSF cells using Lipofectamine 2000. After $48 \mathrm{~h}$, luciferase activity was analyzed by the Dual-Luciferase Assay System (Promega Corporation, Madison, WI, USA), according to the manufacturer's protocols.

Western blotting. HSF cells were transfected with miR-205-5p mimics, NC or miR-205-5p mimic+smad2-plamid for $48 \mathrm{~h}$, then cells were collected and total proteins were extracted in $40 \mathrm{mM}$ Tris- $\mathrm{HCl}$ (pH 7.4) containing $150 \mathrm{mM} \mathrm{NaCl}$ and $1 \%$ (v/v) Triton X-100, supplemented with protease inhibitors. Protein concentration was determined using the bicinchoninic acid protein assay (Pierce; Thermo Fisher Scientific, Inc.). Equal amounts of proteins were resolved on 10\% SDS-PAGE gels, and then transferred to a PVDF membrane (EMD Millipore, Billerica, MA, USA). After blocking with 5\% skimmed milk in TBST, then probed with antibodies against smad2, Col I, Col III, $\beta$-actin (All buy from Cell Signaling Technology, Inc., Danvers, MA, USA). After three times' washing, blots were then incubated with horseradish peroxidase (HRP) conjugated secondary antibodies. Immunoreactive bands were visualized using the enhanced chemiluminescence detection system. The protein levels of the stripes were normalized based on the gray value of $\beta$-actin.

Statistical analysis. SPSS17.0 software was used to analyze the data. Values were expressed as mean \pm SD of experiments performed in triplicate. Data were analyzed by one-way ANOVA or Student's t-test. Statistical significance was defined as $\mathrm{P}<0.05$.

\section{Results}

miR-205-5p is downregulated in HS tissues and HSFs. Fifteen HS and 15 paired normal skin (NS) tissues were recruited in this study. Compared to the normal skin, the expression of miR-205-5p were significantly decreased in HS tissues (Fig. 1A). Meanwhile we detected the expression of miR-205-5p in human embryonic skin fibroblasts (CCC-ESF-1) and human hypertrophic scar fibroblasts (HSFs), the result was consistent 
with tissues, HSFs have lower expression of miR-205-5p (Fig. 1B). Thus, we choose HSF cells for the further study.

miR-205-5p directly targets smad2. Firstly, we use TargetScan to predict the potential targets of miR-205-5p, and about 600 genes were found as the potential target genes of miR-205-5p, including smad2 (Fig. 2A). Smad2, one of the important members of the transforming growth factor $b$ (TGF- $\beta$ ) pathway family which involved in cell growth regulation, plays critical roles in ECM synthesis and degradation. Thus, we choose smad 2 for further analysis. And the luciferase reporter assay showed that the relative luciferase activity in miR-205-5p mimics and smad2 WT 3'UTR reporter co-transfected hHSFs significantly decreased compared with cells co-transfected with mimic control and and smad2 WT 3'UTR reporter (Fig. 2B).

In addition, the expression of smad 2 in HS tissues and HSF cells were detected by qRT-PCR, the data showed that smad2 was significantly upregulated in HS tissues and HSF cells compared with normal tissues or cells (Fig. 3A and B). Compared with miR-205-5p, the approximate reverse pattern of smad2 expression was found.

Furthermore, we transfected HSF cells with miR-205-5p mimics, miR-205-5p mimic+smad2-plasmid or NC. The transfection efficiency was examined by qRT-PCR (Fig. 3C). Overexpression of miR-205-5p markedly decreased the protein level of smad2, and this decrease can be reversed by smad2-plasmid (Fig. 3D). Moreover, we detected the mRNA level of smad2 after transfecting miR-205-5p mimics, miR-205-5p mimic+smad2-plasmid or NC in HSF cells. As expected, overexpression of miR-205-5p downregulated the mRNA level of smad2 in HSFs. However, the effect was eliminated when co-transfected the miR-205-5p mimics with smad2-plasmid (Fig. 3E). These results suggest that miR-205-5p directly targets smad2.

miR-205-5p suppresses HSF cell proliferation. Given our limited understanding of the role played by miR-205-5p in HSF cells, we examined the effects of miR-205-5p gain-of-function on HS formation. After transfection for $48 \mathrm{~h}$, cell proliferation assay was applied. The CCK-8 results showed that miR-205-5p mimics significantly suppressed HSF cell proliferation (Fig. 4). Meanwhile, co-transfected smad2-plasmid with miR-205-5p mimics did not significantly decreased the cell proliferation ability. The data indicate that miR-205-5p suppresses HSF cell proliferation through targeting smad 2 .

miR-205-5p induces HSF cell apoptosis in vitro. HSF cell apoptosis was detected $48 \mathrm{~h}$ after transfected with miR-205-5p mimics, miR-205-5p mimic+smad2-plasmid or NC. Flow cytometry analysis demonstrated that cell apoptosis was increased in miR-205-5p-mimics-transfected HSFs compared to control groups. Moreover, co-transfected smad2-plasmid with miR-205-5p mimics did not notably induced cell apoptosis rate (Fig. 5). Together, these data indicate that miR-205-5p inhibits cell grows and promotes apoptosis of HSF cells.

miR-205-5p mediated suppressive effect on ECM production associated proteins by preventing AKT phosphorylation. To further investigate the molecular mechanism of the
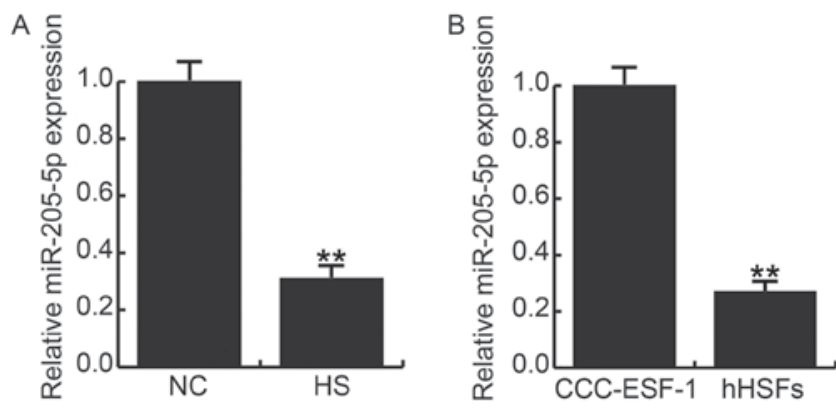

Figure 1. miR-205-5p expression in hypertrophic scar. Relative miR-205-5p level in hypertrophic scar tissues (A) and hHSFs (B) were detected by qRT-PCR. Data were expressed as mean \pm SD. ${ }^{* *} \mathrm{P}<0.01$ vs. NC or CCC-ESF-1.

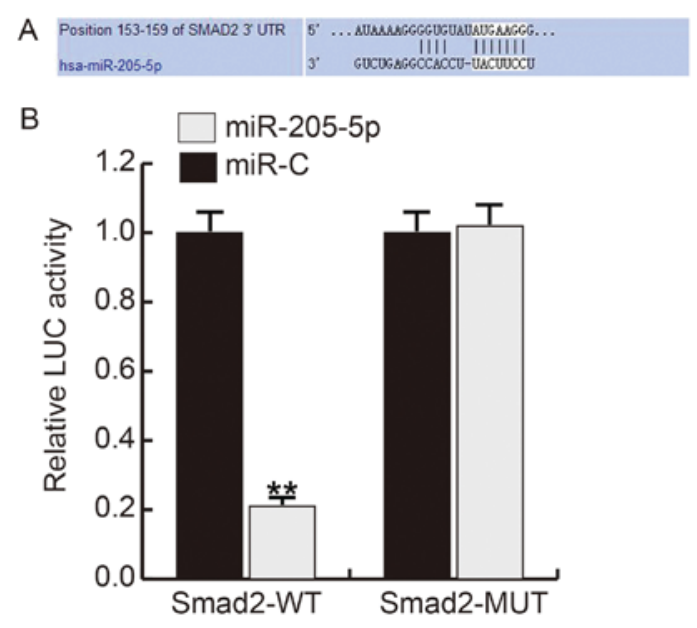

Figure 2. miR-205-5p targets smad2. (A) We used TargetScan to predict the interaction between miR-205-5p and 3'UTR of smad2; (B) Luciferase activity was detected by Dual luciferase assay. Here, 'MUT' indicates the smad2 3 ' UTR with a mutation in the miR-205-5p binding site. UTR, untranslated region. All data are presented as the mean $\pm \mathrm{SD} .{ }^{* *} \mathrm{P}<0.01 \mathrm{vs}$. miR-C.

miR-205-5p effects, we evaluated the role of AKT signaling in miR-205-5p-mediated smad2 in HSF. The phosphorylation level of AKT was examined in HSFs transfected with miR-205-5p mimics, miR-205-5p mimic+smad2-plasmid or NC. Overexpression of miR-205-5p decreased AKT phosphorylation. However, co-transfected smad2-plasmid with miR-205-5p mimics could eliminate the changes caused by miR-205-5p mimics (Fig. 6). Thus, the miR-205-5p inhibited AKT signaling pathway was activated by smad2 overexpression. In addition, mRNA and protein expression of $\alpha$-SMA, Col I and Col III were downregulated in miR-205-5p mimics transfected HSFs, and the smad2-plasmid significantly counteracted this effect (Fig. 7). Taken together, these data indicate that miR-205-5p suppresses AKT phosphorylation, downregulates Col I, Col III, and $\alpha$-SMA in HSFs through smad2 mediator.

\section{Discussion}

The regulation of wound healing and its disorders in HS are complex and incomplete $(25,26)$. Histologically, HS is characterized by increased myofibroblasts and mast cells, hypervascularity, excessive extracellular matrix (ECM) (24). 


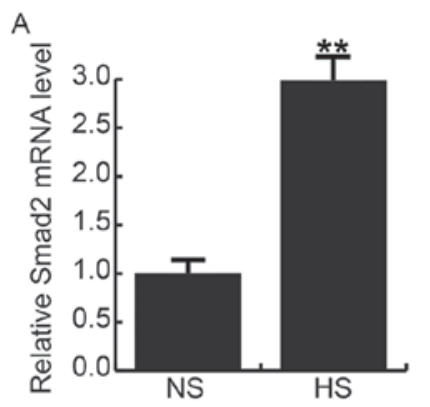

D

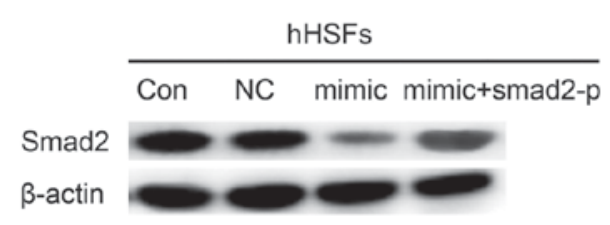

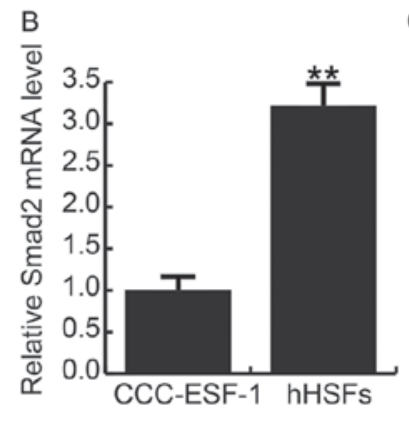
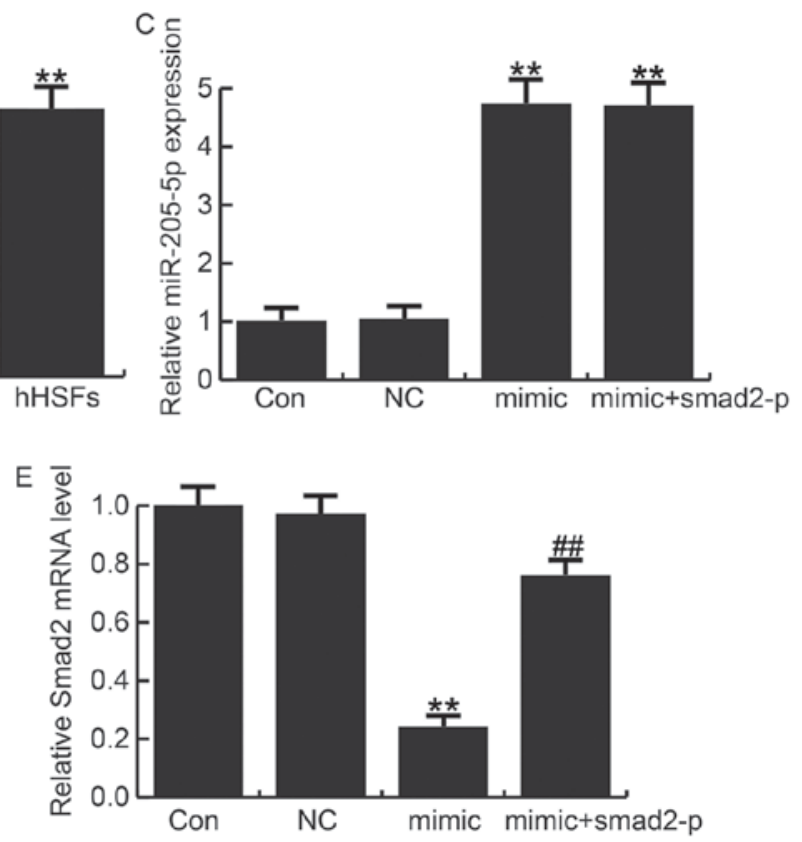

Figure 3. Effects of miR-205-5p on smad2 expression in hHSFs. (A and B) relative smad2 mRNA expression in hypertrophic scar tissues/hHSFs was determined by QRT-PCR. (C) relative miR-205-5p in different groups; (D) effect of miR-205-5p on smad2 protein expression in hHSFs; (E) effect of miR-205-5p on smad 2 mRNA expression in hHSFs. Con, control group, cells without any treatment; NC, negative control group, cells transfected with the negative control vector; mimic, cells transfected with miR-205-5p mimics; mimic+smad2-p, cells co-transfected with miR-205-5p mimics and smad2-plasmid. Data are displayed as the mean \pm SD. ${ }^{* *} \mathrm{P}<0.01$ vs. NS, CCC-ESF-1 or Con; ${ }^{\# \#} \mathrm{P}<0.01$ vs. mimic.

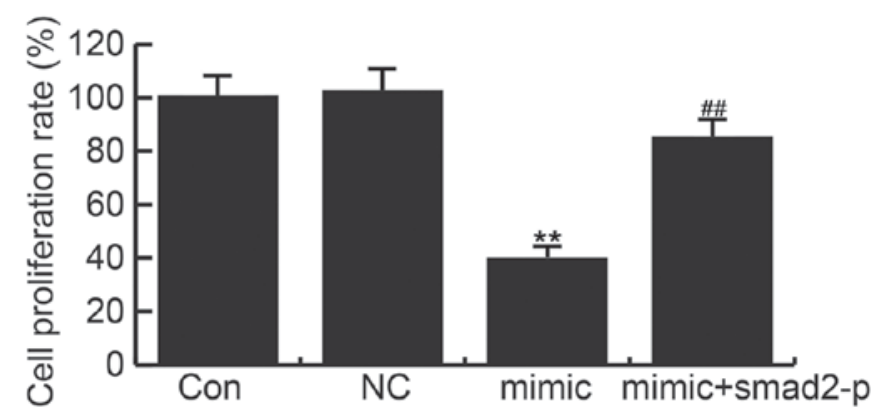

Figure 4. Effects of miR-205-5p on hHSFs cell proliferation. $48 \mathrm{~h}$ after cell transfection, CCK-8 assay was used to detect the effect of miR-205-5p on hHSFs cell proliferation. Con, control group, cells without any treatment; $\mathrm{NC}$, negative control group, cells transfected with the negative control vector; mimic, cells transfected with miR-205-5p mimics; mimic+smad2-p, cells co-transfected with miR-205-5p mimics and smad2-plasmid. Data are displayed as the mean $\pm \mathrm{SD} .{ }^{* *} \mathrm{P}<0.01$ vs. Con; ${ }^{\# \#} \mathrm{P}<0.01$ vs. mimic.

Unfortunately, the current treatment for HS has limited efficacy (27). Abnormal expression of miRNAs may contribute significantly to the progression of skin fibrosis (28). miR-205-5p, a functional miRNA, has received much attention from researchers in recent years. The above studies suggested that miR-205-5p acts either as an oncogene or tumor suppressor gene, depending on the cellular environment. Our preliminary data show that miR-205-5p is significantly reduced in HS tissues and HSF cells compared to normal tissues and cells. Also, we found that the expression of smad 2 was negatively correlated with miR-205-5p. Therefore, we hypothesis smad2 gene was associated with miR-205-5p.

In order to confirm this hypothesis, firstly we use TargetScan to predict the potential targets of miR-205-5p.
Smad2 was identified as a potential miR-205-5p target gene. In addition, luciferase reporter assay verified miR-205-5p directly binding to the 3 '-UTR of $\operatorname{smad} 2$. Moreover, the expression of smad 2 in HS tissues and HSF cells were significantly upregulated, smad 2 expression was the opposite pattern compared to miR-205-5p. Overexpression of miR-205-5p could downregulate smad 2 expression. These results suggest that miR-205-5p directly targets smad2.

Smad2 is a tumor suppressor that belongs to the receptor-activated SMAD family (29). Previous studies have revealed that upregulation of smad 2 suppressed TGF- $\beta$ induced EMT and cell motility and invasion. In this study, hHSFs transfected with miR-205-5p mimics showed obvious suppression effect in cell proliferation, and cell apoptosis increased significantly. Co-transfected smad2-plasmid with miR-205-5p mimics could eliminate the effect of miR-205-5p mimics. Further confirmed that miR-205-5p played a role through regulating smad2.

Collagen is the most important extracellular matrix structural protein, and 28 different types of collagen has been identified. Among these types, type 1, 2 and 3 are the most abundant collagen (30). A large number of collagen synthesis and changes are considered to be the main features of HS formation. In previous studies, the expression of Col I and Col III in HSF cells and HS tissues was significantly higher than that in normal cells and healthy tissues (31). Fibroblasts, especially Col I and Col III overdose are responsible for keloid and hypertrophic scar formation. $\alpha$-smooth muscle actin ( $\alpha$-SMA) has been shown to be a key regulator of extracellular matrix metabolism in many tissues (32). In our study, the protein expression of Col I, Col III was significantly reduced in miR-205-5p mimics transfected HSFs by targeting smad2, suggesting that miR-205-5p works as an anti-fibrotic factor in HSFs. 

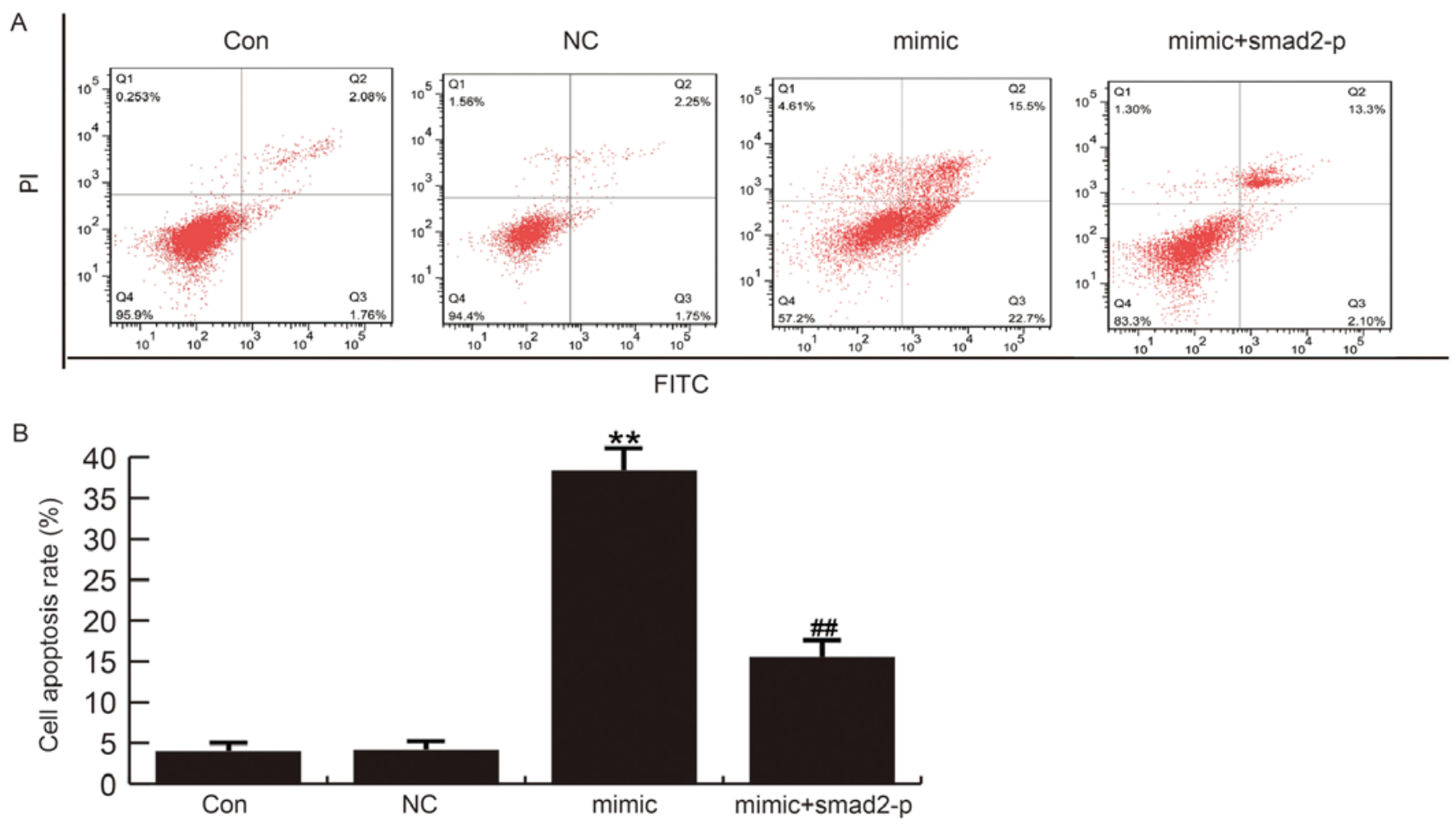

Figure 5. Effects of miR-205-5p on hHSFs cell apoptosis. (A) At $48 \mathrm{~h}$ after cell transfection, effect of miR-205-5p on hHSFs cell apoptosis was analyzed by using Flow Cytometry. Con, control group, cells without any treatment; NC, negative control group, cells transfected with the negative control vector; mimic, cells transfected with miR-205-5p mimics; mimic+smad2-p, cells co-transfected with miR-205-5p mimics and smad2-plasmid. (B) Data are displayed as the mean \pm SD. ${ }^{* *} \mathrm{P}<0.01$ vs. Con; ${ }^{\# \#} \mathrm{P}<0.01$ vs. mimic.

A

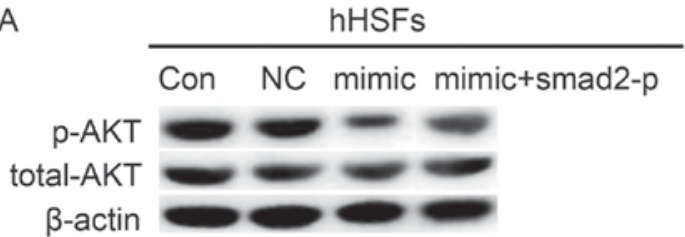

B

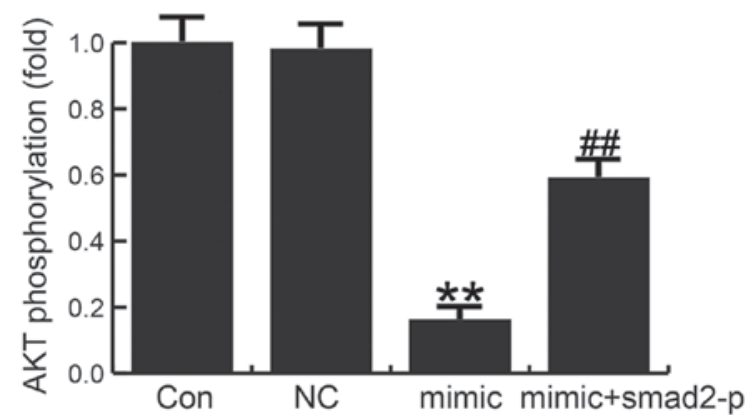

Figure 6. Effects of miR-205-5p on AKT activation in hHSFs. At $48 \mathrm{~h}$ after cell transfection, the protein level of p-AKT was detected by western blotting. (A) The expression levels of AKT phosphorylation were expressed as fold of the control. (B) Con, control group, cells without any treatment; NC, negative control group, cells transfected with the negative control vector; mimic, cells transfected with miR-205-5p mimics; mimic+smad2-p, cells co-transfected with miR-205-5p mimics and smad2-plasmid. Data are displayed as the mean \pm SD. ${ }^{* *} \mathrm{P}<0.01$ vs. Con; ${ }^{\# \#} \mathrm{P}<0.01$ vs. mimic.

PI3K/AKT is mediated by multifunctional signaling pathways in cell proliferation, motility, differentiation, fibrosis and lipid metabolism. Previous studies have shown that AKT signaling plays a key role in the development of fibrosis associated with diabetic nephropathy (33). Our results indicated that the AKT signaling pathway was inhibited by miR-205-5p mimics; however, the smad2-plasmid significantly counteracted the effects of miR-205-5p mimics. In addition, the downregulated expression of ColI, Col III, and $\alpha$-SMA caused by miR-205-5p mimics was also eliminate by smad2-plasmid. Taken together, these results indicate that miR-205-5p mediates HS formation, partly due to AKT inhibition by targeting smad2.

In conclusion, our study shows that miR-205-5p inhibits HSF cell proliferation and promotes apoptosis and reduces the expression of ECM-related proteins through inhibiting AKT pathway by directly targeting smad 2 , thus affecting HS formation. Thus, this study provided evidence to determine that miR-205-5p may be a useful target for the treatment of HS. However, as age and tissue location are important factors when assessing the degree of healing of the scar tissue, our research may have some limitations, thus, further researches are needed to prove our conclusion. We will study the relationship between miR-205-5p expression and the age of patient or tissue location, and further explore the role of miR-205-5p in HS formation in the future.

\section{Acknowledgements}

Not applicable. 
A

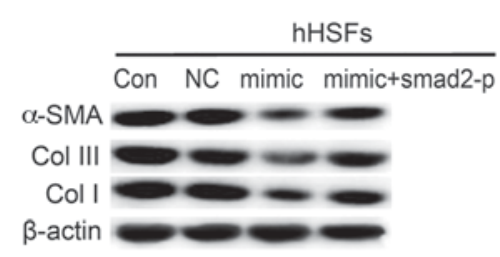

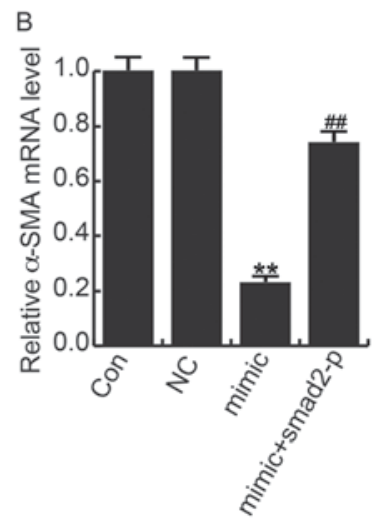

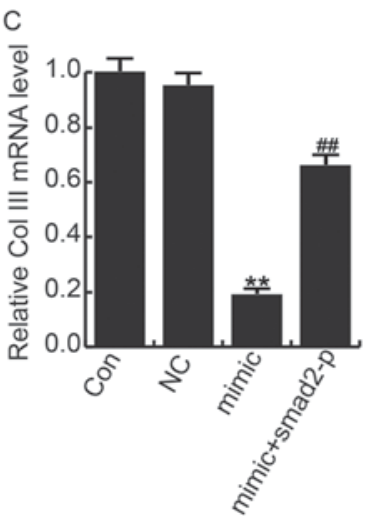

Figure 7. Effects of miR-205-5p on $\alpha$-SMA, Col I and Col III expression in hHSFs. At 48 h after cell transfection, $\alpha$-SMA, collagen I and collagen III expression were determined by western blotting (A) and qRT-PCR (B-D) respectively. Con: control group, cells without any treatment; NC: negative control group, cells transfected with the negative control vector; mimic: cells transfected with miR-205-5p mimics; mimic+smad2-p: cells co-transfected with miR-205-5p mimics and smad2-plasmid. Data are displayed as the mean $\pm \mathrm{SD} .{ }^{* *} \mathrm{P}<0.01$ vs. Con; ${ }^{\# \#} \mathrm{P}<0.01$ vs. mimic.

\section{Funding}

No funding was received.

\section{Availability of data and materials}

The analyzed data sets generated during the present study are available from the corresponding author on reasonable request.

\section{Authors' contributions}

JQ and YW designed the study. JQ, YL, KH, YZ and XZ were responsible for data access and analysis. All authors collaborated to interpret the results and develop the manuscript.

\section{Ethics approval and consent to participate}

All the protocols were approved by the Ethics Committee of the Affiliated Hospital of Nantong University (Nantong, China), and every patient provided informed consent.

\section{Patient consent for publication}

All patients provided informed consent for publication.

\section{Competing interests}

The authors declare that they have no competing interests.

\section{References}

1. Aarabi S, Bhatt KA, Shi Y, Paterno J, Chang EI, Loh SA, Holmes JW, Longaker MT, Yee H and Gurtner GC: Mechanical load initiates hypertrophic scar formation through decreased cellular apoptosis. FASEB J 21: 3250-3261, 2007.

2. van der Veer WM, Bloemen MC, Ulrich MM, Molema G, van Zuijlen PP, Middelkoop E and Niessen FB: Potential cellular and molecular causes of hypertrophic scar formation. Burns 35: 15-29, 2009.

3. Younai S, Nichter LS, Wellisz T, Reinisch J, Nimni ME and Tuan TL: Modulation of collagen synthesis by transforming growth factor-beta in keloid and hypertrophic scar fibroblasts. Ann Plast Surg 33: 148-154, 1994.
4. Shi-Wen X, Leask A and Abraham D: Regulation and function of connective tissue growth factor/CCN2 in tissue repair, scarring and fibrosis. Cytokine Growth Factor Rev 19: 133-144, 2008.

5. Kashiyama K, Mitsutake N, Matsuse M, Ogi T, Saenko VA, Ujifuku K, Utani A, Hirano A and Yamashita S: miR-196a downregulation increases the expression of type I and III collagens in keloid fibroblasts. J Invest Dermatol 132: 1597-1604, 2012.

6. Li P, He QY and Luo CQ: Overexpression of miR-200b inhibits the cell proliferation and promotes apoptosis of human hypertrophic scar fibroblasts in vitro. J Dermatol 41: 903-911, 2014.

7. Hammond SM: An overview of microRNAs. Adv Drug Deliv Rev 87: 3-14, 2015.

8. Wilson RC and Doudna JA: Molecular mechanisms of RNA interference. Annu Rev Biophys 42: 217-239, 2013.

9. Sun W, Julie Li YS, Huang HD, Shyy JY and Chien S: microRNA: A master regulator of cellular processes for bioengineering systems. Annu Rev Biomed Eng 12: 1-27, 2010.

10. Guo H, Ingolia NT, Weissman JS and Bartel DP: Mammalian microRNAs predominantly act to decrease target mRNA levels. Nature 466: 835-840, 2010.

11. Flynt AS and LaiEC: Biological principles of microRNA-mediated regulation: Shared themes amid diversity. Nat Rev Genet 9: 831-842, 2008.

12. Miska EA: How microRNAs control cell division, differentiation and death. Curr Opin Genet Dev 15: 563-568, 2005.

13. Bushati N and Cohen SM: microRNA functions. Annu Rev Cell Dev Biol 23: 175-205, 2007.

14. Liang P, Lv C, Jiang B, Long X, Zhang P, Zhang M, Xie T and Huang X: MicroRNA profiling in denatured dermis of deep burn patients. Burns 38: 534-540, 2012.

15. Bi S, Chai L, Yuan X, Cao C and Li S: MicroRNA-98 inhibits the cell proliferation of human hypertrophic scar fibroblasts via targeting Col1A1. Biol Res 50: 22, 2017.

16. Xiao K, Luo X, Wang X and Gao Z: MicroRNA-185 regulates transforming growth factor- $\beta 1$ and collagen- 1 in hypertrophic scar fibroblasts. Mol Med Rep 15: 1489-1496, 2017.

17. Li G, Zhou R, Zhang Q, Jiang B, Wu Q and Wang C: Fibroproliferative effect of microRNA-21 in hypertrophic scar derived fibroblasts. Exp Cell Res 345: 93-99, 2016.

18. Zeng Y, Zhu J, Shen D, Qin H, Lei Z, Li W, Liu Z and Huang JA: MicroRNA-205 targets SMAD4 in non-small cell lung cancer and promotes lung cancer cell growth in vitro and in vivo. Oncotarget 8: 30817-30829, 2017.

19. Nguyen-Vu T, Wang J, Mesmar F, Mukhopadhyay S, Saxena A, McCollum CW, Gustafsson JA, Bondesson M and Williams C: Estrogen receptor beta reduces colon cancer metastasis through a novel miR-205-PROX1 mechanism. Oncotarget 7: 42159-42171, 2016.

20. Tucci P, Agostini M, Grespi F, Markert EK, Terrinoni A, Vousden KH, Muller PA, Dötsch V, Kehrloesser S, Sayan BS, et al: Loss of p63 and its microRNA-205 target results in enhanced cell migration and metastasis in prostate cancer. Proc Natl Acad Sci USA 109: 15312-15317, 2012. 
21. Pan F, Mao H, Bu F, Tong X, Li J, Zhang S, Liu X, Wang L, Wu L, Chen R, et al: Spl-mediated transcriptional activation of miR-205 promotes radioresistance in esophageal squamous cell carcinoma. Oncotarget 8: 5735-5752, 2017.

22. Jiang M, Zhong T, Zhang W, Xiao Z, Hu G, Zhou H and Kuang H: Reduced expression of miR-205-5p promotes apoptosis and inhibits proliferation and invasion in lung cancer A549 cells by upregulation of ZEB2 and downregulation of erbB3. Mol Med Rep 15: 3231-3238, 2017.

23. Jang SJ, Choi IS, Park G, Park G, Moon DS, Choi JS, Nam MH, Yoon SY, Choi CH and Kang SH: MicroRNA-205-5p is upregulated in myelodysplastic syndromes and induces cell proliferation via PTEN suppression. Leuk Res 47: 172-177, 2016.

24. Tredget EE, Nedelec B, Scott PG and Ghahary A: Hypertrophic scars, keloids, and contractures. The cellular and molecular basis for therapy. Surg Clin North Am 77: 701-730, 1997.

25. Armour A, Scott PG and Tredget EE: Cellular and molecular pathology of HTS: Basis for treatment. Wound Repair Regen 15 (Suppl 1): S6-S17, 2007.

26. Schäfer $M$ and Werner S: Transcriptional control of wound repair. Annu Rev Cell Dev Biol 23: 69-92, 2007.

27. Kwan P, Hori K, Ding J and Tredget EE: Scar and contracture: Biological principles. Hand Clin 25: 511-528, 2009.

28. Babalola O, Mamalis A, Lev-Tov H and Jagdeo J: The role of microRNAs in skin fibrosis. Arch Dermatol Res 305: 763-776, 2013.

29. Syed V: TGF- $\beta$ signaling in cancer. J Cell Biochem 117: $1279-1287,2016$
30. Volk SW, Wang Y, Mauldin EA, Liechty KW and Adams SL: Diminished type III collagen promotes myofibroblast differentiation and increases scar deposition in cutaneous wound healing. Cells Tissues Organs 194: 25-37, 2011

31. Zhou R, Zhang Q, Zhang Y, Fu S and Wang C: Aberrant miR-21 and miR-200b expression and its pro-fibrotic potential in hypertrophic scars. Exp Cell Res 339: 360-366, 2015

32. Satish L, Gallo PH, Baratz ME, Johnson S and Kathju S: Reversa of TGF- $\beta 1$ stimulation of $\alpha$-smooth muscle actin and extracellular matrix components by cyclic AMP in Dupuytren's-derived fibroblasts. BMC Musculoskelet Disord 12: 113, 2011.

33. Lu Q, Zuo WZ, Ji XJ, Zhou YX, Liu YQ, Yao XQ, Zhou XY, Liu YW, Zhang F and Yin XX: Ethanolic Ginkgo biloba leaf extract prevents renal fibrosis through Akt/mTOR signaling in diabetic nephropathy. Phytomedicine 22: 1071-1078, 2015.

(i)(3) This work is licensed under a Creative Commons EY NG ND Attribution-NonCommercial-NoDerivatives 4.0 International (CC BY-NC-ND 4.0) License. 